\title{
Adaptive Barrier Control for Nonlinear Servomechanisms with Friction Compensation
}

\author{
Shubo Wang $\mathbb{D},{ }^{1}$ Haisheng Yu $\mathbb{D},{ }^{1}$ Xuehui Gao $\mathbb{D}^{2},{ }^{2}$ and Na Wang $\mathbb{D}^{1}$ \\ ${ }^{1}$ College of Automation and Electrical Engineering, Qingdao University, Qingdao 266071, China \\ ${ }^{2}$ Department of Mechanical and Electrical Engineering, Shandong University of Science and Technology, Tai'an 2701019, China
}

Correspondence should be addressed to Shubo Wang; wangshubo1130@126.com

Received 24 March 2018; Revised 29 April 2018; Accepted 3 May 2018; Published 11 July 2018

Academic Editor: Liang $\mathrm{Hu}$

Copyright (C) 2018 Shubo Wang et al. This is an open access article distributed under the Creative Commons Attribution License, which permits unrestricted use, distribution, and reproduction in any medium, provided the original work is properly cited.

\begin{abstract}
This paper proposes an adaptive barrier controller for servomechanisms with friction compensation. A modified LuGre model is used to capture friction dynamics of servomechanisms. This model is incorporated into an augmented neural network (NN) to account for the unknown nonlinearities. Moreover, a barrier Lyapunov function (BLF) is utilized to each step in a backstepping design procedure. Then, a novel adaptive control method is well suggested to ensure that the full-state constraints are within the given boundary. The stability of the closed-loop control system is proved using Lyapunov stability theory. Comparative experiments on a turntable servomechanism confirm the effectiveness of the devised control method.
\end{abstract}

\section{Introduction}

The high-performance modeling and motion control of servomechanisms have been of great importance in practical engineering application and have also drawn significant attention in academic fields [1-6] due to their compact structure and efficiency. Nevertheless, the nonsmooth dynamics, including nonlinear friction [7-9] and dead-zone [10-12], introduced by transmission devices may deteriorate the control performance. To reduce the effect of the nonlinear friction, various control algorithms have been proposed such as sliding mode technique (SMC) [13-18] and adaptive control [19-23]. In addition, intelligent control methods (e.g., fuzzy logic systems (FLS) [24-26] and neural networks (NN) [27-29]) have also been utilized to approximate the nonlinearities using their learning abilities. For example, an adaptive prescribed performance tracking control was developed for servomechanisms to achieve position tracking [28]. In [26], an adaptive fuzzy control based on command filter was proposed for nonlinear systems, and an adaptive neural network control method was devised for permanent magnet synchronous motor (PMSM) system [19].

Moreover, the unknown disturbances can also deteriorate the control precision of servomechanisms. To overcome this issue, an effective method is to design a disturbance observer (DOB) to estimate the unknown disturbances [30-32]. In [31], Ohnishi proposed a DOB-based control method. Then, the disturbance observer technique-based control methods were widely utilized in practice. In [32], a neural network disturbance observer (NNDOB) was developed for servosystem to estimate the unknown dynamics. In [33], a nonlinear disturbance observer (NDOB) was devised for robotic manipulators to compensate for the unknown friction. Recently, Han proposed a new disturbance estimation technique named extended state observer (ESO) [34]. The main feature is that the ESO can not only estimate the unmeasured system states but also observe the unknown disturbances. The ESO has been widely applied in many control fields [35-39]. In [40], an ESO was employed to estimate mismatched disturbances of power converter systems. In [41], an ESO was used to observe state vectors and system uncertainties and an adaptive controller was designed using the feedback linearization method for a robotic system. In [42], to estimate unknown disturbances and system uncertainties, an event-triggered active disturbance rejection control (ADRC) was proposed for physical servosystems. In [1], an ESO based on an adaptive funnel control scheme was developed to achieve position tracking control of 
servomechanisms, where the ESO was used to estimate the unknown disturbance and system states. Although the aforementioned control methods can improve the control performance of the servosystem, it is noted that the transient performance can not be guaranteed.

In recent years, a new constraint function called prescribed performance function (PPC) was proposed by Rovithakis et al. in $[43,44]$. The main feature of this technique is to provide the prescribed performance function (PPF) so that the tracking error of an original nonlinear system can be transformed into a new error of a transformed system. Then, the tracking error can be guaranteed within a given prescribed boundary. The PPC method has been widely applied in practice such as vehicle active suspension systems [45, 46], unmanned surface vehicle systems [47], nonlinear systems [48, 49], fault-tolerant control [50], and robot manipulators [51]. On the other hand, the barrier Lyapunov function (BLF) is used as a constraint control which is utilized to transform the error into a new error without constraint [52]. In [53], a boundary controller with BLF was proposed for flexible marine riser to suppress the riser vibration. In [54], a BLF is used to constraint the error and integrated into the adaptive backstepping control design to guarantee the states within the barrier. A BLF-based learning control was presented for hypersonic flight vehicle with AOA constraint and actuator faults [55]. In [56], an adaptive BLF controller was devised for PMSM with full-state constraints. An adaptive neural control strategy was designed for multiple input multiple output (MIMO) nonlinear systems with various constraints [57].

This paper proposes an adaptive barrier control method for servomechanisms with friction compensation. A modified continuous friction model is developed to capture the friction dynamics. The NN is employed to estimate unknown dynamics (e.g., nonlinear friction, external disturbances, and system uncertainties) and is incorporated into an adaptive controller to reduce the effect of unknown dynamics. Moreover, a BLF is introduced to each step in a backstepping design procedure to improve the control performance. Then, a new adaptive controller is devised using a recurrent feedback form to ensure the states within the given constraints. The semiglobal boundedness of all closed-loop signals is ensured, and the tracking error converges to a neighborhood of zero. Finally, the effectiveness of the proposed control method is validated via experimental results.

The main contributions are summarized:

(1) A new friction model is presented by using the modified LuGre friction model to describe the nonlinear friction. The $\mathrm{NN}$ is used to approximate friction dynamics and unknown disturbances of servomechanisms.

(2) To improve the control performance, a BLF is used to transform the error into a new error without constraint, a novel adaptive neural backstepping design procedure is designed, and the new-type adaptation law is developed. We prove that all signals of the closed-loop system are bounded and the tracking error can converge to a small region.

(3) Extensive comparative experiment results provide evidence of the advantage of suggestion approach in comparison to adaptive neural dynamic surface controller (ANDSC) and PID.

The remainder of this brief is organized as follows. System model is given in Section 2. Controller design is provided in Section 3, and Section 4 presents system stability analysis. Experiment results are shown in Section 5 and Section 6 describes the conclusions.

\section{Preliminaries and Problem Statement}

2.1. System Model. Considering the motion tracking control of a class of nonlinear servomechanisms [39] (see Figure 1), the dynamic mathematical model of such system can be described as

$$
\begin{aligned}
\left(\begin{array}{c}
\dot{i}_{d} \\
\dot{i}_{q} \\
\ddot{q}
\end{array}\right)= & \left(\begin{array}{ccc}
\frac{-R}{L} & -n_{\mathrm{p}} \dot{q} & 0 \\
-n_{\mathrm{p}} \dot{q} & \frac{-R}{L} & \frac{n_{\mathrm{p}} \psi_{\mathrm{f}}}{L} \\
0 & \frac{K_{\mathrm{T}}}{J} & \frac{1}{J}
\end{array}\right)+\left(\begin{array}{c}
i_{d} \\
i_{q} \\
\dot{q}
\end{array}\right) \\
& +\left(\begin{array}{c}
\frac{u_{d}}{L} \\
\frac{u_{q}}{L} \\
\frac{-T_{\mathrm{f}}-T_{1}-T_{\mathrm{d}}-f(q, \dot{q})}{J}
\end{array}\right),
\end{aligned}
$$

where $i_{d}$ and $i_{q}$ are the $d$-axis and $q$-axis stator voltages, respectively; $u_{d}$ and $u_{q}$ are the $d$-axis and $q$-axis stator currents, respectively; $n_{\mathrm{p}}$ is the number of pole pairs; $R$ and $L$ are the stator resistance and stator inductance, respectively; $T_{1}$ is the load torque; $K_{\mathrm{T}}$ is the torque constant; $T_{\mathrm{d}}$ is the disturbance torque; $\psi_{\mathrm{f}}$ is the rotor flux linkage; $q$ is the angular position; $\dot{q}$ is the angular speed; $T_{\mathrm{f}}$ is the friction torque; $J$ is the motor inertia; and $f(q, \dot{q})$ stands for the unknown resonances and uncertainties.

In practice, the parameter $L / R$ is smaller than the mechanical time constant. Thus, the parameter $L d i_{q} / d t$ decays very rapidly to zero. In addition, to eliminate the couplings between the angular speed and current, the $d$-axis reference current $i_{d}^{*}$ is set to zero. In this case, (1) can be simplified as

$$
\left(\begin{array}{c}
\dot{i}_{q} \\
\ddot{q}
\end{array}\right)=\left(\begin{array}{cc}
\frac{-R}{L} & \frac{K_{E}}{L} \\
\frac{K_{\mathrm{T}}}{J} & \frac{1}{J}
\end{array}\right)\left(\begin{array}{l}
i_{q} \\
\dot{q}
\end{array}\right)+\left(\begin{array}{c}
\frac{u_{d}}{L} \\
\frac{-T_{\mathrm{f}}-T_{1}-T_{\mathrm{d}}-f(q, \dot{q})}{J}
\end{array}\right),
$$




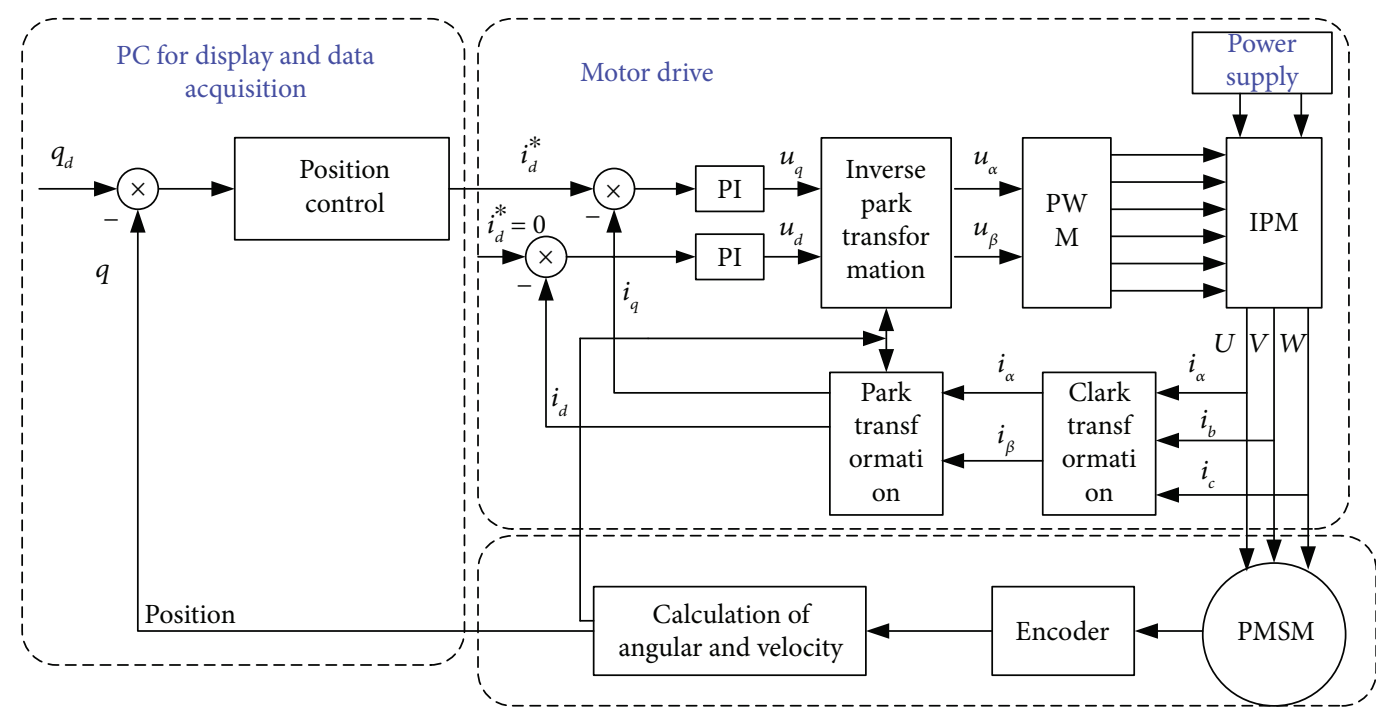

FIGURE 1: Schematic diagram of position control for PMSM.

where $K_{E}=n_{\mathrm{p}} \psi_{\mathrm{f}}$.

Choose the state vector $\mathbf{x}=\left[x_{1}, x_{2}\right]^{T}=[q, \dot{q}]^{T}$; then, the (2) can be simplified as

$$
\begin{aligned}
& \dot{x}_{1}=x_{2}, \\
& \dot{x}_{2}=\frac{1}{J}\left(K_{1} u-K_{2} x_{2}-f\left(x_{1}, x_{2}\right)-T_{\mathrm{d}}-T_{1}-T_{\mathrm{f}}\right), \\
& y=x_{1},
\end{aligned}
$$

where $K_{1}=K_{\mathrm{T}} / R, K_{2}=K_{\mathrm{T}} K_{E} / R$ are positive constants and $u=u_{d}$ is the control input voltage.

2.2. Modified LuGre Friction Model. Over the past years, various friction models have been proposed to describe the friction dynamics. Among these models, the LuGre model is widely used to capture the friction behaviors. To describe the friction dynamics of servomechanisms, the LuGre model is described by

$$
T_{f}=\sigma_{0} z+\sigma_{1} \dot{z}_{1}+\sigma_{2} \omega
$$

where $\sigma_{0}, \sigma_{1}$, and $\sigma_{2}$ are the friction coefficients; $\omega$ denotes the angular velocity; and $z$ is the unmeasurable internal friction state which can be represented by

$$
\dot{z}=\omega\left[1-\frac{1}{g(w)} z\right]
$$

where $g(w)$ is the nonlinear function. Usually, the nonlinear function is given as

$$
g(\omega)=\left[f_{\mathrm{c}}+\left(f_{\mathrm{s}}-f_{\mathrm{c}} e^{-\omega / \omega_{\mathrm{s}}}\right)^{2}\right] \operatorname{sgn}(\omega),
$$

where $f_{\mathrm{c}}$ denotes the coulomb friction and $f_{\mathrm{s}}$ is the stiction force; $\omega_{\mathrm{s}}$ is the Stribeck velocity.

It is worth to note that the Stribeck function $g(\omega)$ is a discontinuous function due to signum function in $g(\omega)$. The conventional LuGre model can not be used in the backstepping controller design. To overcome this issue, we will employ a modified Stribeck function $g(\omega)$ in this paper. Hence, the modified nonlinear function $g(\omega)$ [58] is given as

$$
g(\omega)=\left(f_{\mathrm{s}}-f_{\mathrm{c}}\right)\left[\tanh \left(c_{1} \omega-\tanh \left(c_{2} \omega\right)\right)\right]+f_{\mathrm{c}} \tanh \left(c_{3} \omega\right),
$$

where $c_{1}, c_{2}$, and $c_{3}$ are the positive constants.

Defining a new function $N(\omega)=\omega / g(\omega)$, the modified model can be written as

$$
\begin{aligned}
T_{f} & =\sigma_{0} z+\sigma_{1} \dot{z}_{1}+\sigma \omega, \\
\dot{z} & =\omega-N(\omega) z .
\end{aligned}
$$

When $\dot{z}=0$, the stead-steady friction $f_{\text {ss }}$ can be obtained

$$
\begin{aligned}
f_{\mathrm{ss}}= & \sigma_{0}\left(f_{\mathrm{s}}-f_{\mathrm{c}}\right)\left[\tanh \left(c_{1} \omega-\tanh \left(c_{2} \omega\right)\right)\right] \\
& +\sigma_{0} f_{\mathrm{c}} \tanh (c 3 \omega)+\sigma_{2} \omega .
\end{aligned}
$$

2.3. NN Approximation. NNs have been widely used in modeling and control of nonlinear functions using their approximations and learn abilities [59-61]. In this paper, the $\mathrm{NN}$ is employed to approximate a continuous function $f(x) . f(\mathbf{x}): R^{m} \rightarrow R$ over a compact domain $\Omega \in R^{m}$ is defined as

$$
f(\mathbf{x})=W_{0}^{*} X(\mathbf{x})+\varepsilon^{*} \quad \forall \mathbf{x} \in \Omega \subset R^{m},
$$

where $\varepsilon^{*}$ denotes the approximation error of $\mathrm{NN}$ and $\left|\varepsilon^{*}\right| \leq \varepsilon_{m} ; W_{0}^{*}$ is the ideal value of $\mathrm{NN}$ weights that minimizes the approximation error $\varepsilon^{*}$. Therefore,

$$
W_{0}^{*}=\arg \min _{W_{0} \in R^{L}}\left\{\sup _{\mathbf{x} \in \Omega}\left|f(\mathbf{x})-W_{0}^{* T} X(\mathbf{x})\right|\right\} .
$$

Because the ideal NN weight $W_{0}^{*}$ is unknown, we can only use the estimation value $\widehat{W}_{0}^{*}$ of $W_{0}^{*}$ in the control design, which can be updated online via an adaptive law. 


\section{Controller Design}

3.1. Barrier Function. Barrier Lyapunov function (BLF) is used as constraint control, which has been widely used in some fields [55-57]. In this paper, we will adopt the following BLF candidate [52]:

$$
V_{1}=\frac{1}{2} \log \frac{k_{b 1}^{2}}{k_{b 1}^{2}-z_{1}^{2}}
$$

where $\log (\cdot)$ represents the natural logarithm of $\cdot$ and $k_{b 1}$ is the constraint on $z_{1}$, that is, $\left|z_{1}\right|<k_{b 1}$ (see Figure 2). Then, we have the following Lemma 1 .

Lemma 1 [52]. For any positive constant $k_{b 1}$, the following inequality holds for all $z_{1}$ in the interval $\left|z_{1}\right| \leq k_{b 1}$

$$
\log \frac{k_{b 1}^{2}}{k_{b 1}^{2}-z_{1}^{2}}<\frac{z_{1}^{2}}{k_{b 1}^{2}-z_{1}^{2}} .
$$

Assumption 1. The desired trajectory $x_{\mathrm{d}}$ and its first- and second-time derivatives exist and satisfy $\left|x_{d}\right| \leq A_{0} \leq k_{c 1},\left|\dot{x}_{d}\right|$ $\leq A_{1}$ and $\left|\ddot{x}_{d}\right| \leq A_{2}$, where $k_{c 1}, A_{0}$, and $A_{2}$ are positive constants.

3.2. Adaptive Controller Design. In this section, an adaptive barrier controller will be designed using the backstepping technique for servomechanisms with error constraints to achieve position tracking. The control structure is shown in Figure 3. The design steps are given as follows.

Step 1. The tracking error is defined as $z_{1}=x_{1}-x_{d}$, where $x_{d}$ represents the reference signal. Thus, the derivative of $z_{1}$ is

$$
\dot{z}_{1}=\dot{x}_{1}-\dot{x}_{d}=x_{2}-\dot{x}_{d}
$$

Select a BLF as

$$
V_{1}=\frac{1}{2} \log \left(\frac{k_{b 1}^{2}}{k_{b 1}^{2}-z_{1}^{2}}\right)
$$

where $k_{b 1}$ is a design parameter and $k_{b 1}=k_{c 1}-A_{0}$.

Defining the second error surface $z_{2}=x_{2}-\alpha_{1}$, the time derivative of $V_{1}$ is computed by

$$
\dot{V}_{1}=\frac{z_{1}}{k_{b 1}^{2}-z_{1}^{2}} \dot{z}_{1}=\frac{z_{1}}{k_{b 1}^{2}-z_{1}^{2}}\left(z_{2}+\alpha_{1}-\dot{x}_{d}\right) .
$$

The virtual controller $\alpha_{1}$ is designed as

$$
\alpha_{1}=-\lambda_{1} z_{1}+\dot{x}_{d}
$$

where $\lambda_{1}$ is the design parameter.

Then, (15) can be written as

$$
\dot{V}_{1}=-\lambda_{1} \frac{z_{1}}{k_{b 1}^{2}-z_{1}^{2}} z_{1}+\frac{z_{1}}{k_{b 1}^{2}-z_{1}^{2}} z_{2} .
$$

Step 2. The time derivative of $z_{2}$ is

$$
\dot{z}_{2}=\dot{x}_{2}-\dot{\alpha}_{1}
$$

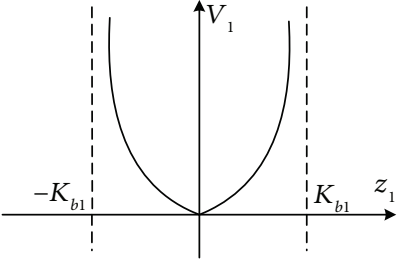

FIgURE 2: Schematic illustration of barrier functions.

Substituting the second equation of system (3) into (19), we have

$$
\begin{aligned}
\dot{z}_{2} & =\frac{1}{J}\left(K_{1} u-K_{2} x_{2}-f\left(x_{1}, x_{2}\right)-T_{\mathrm{d}}-T_{1}-T_{\mathrm{f}}\right)-\dot{\alpha}_{1} \\
& =\frac{1}{J}\left(K_{1} u-F(\mathbf{x})\right)-\dot{\alpha}_{1},
\end{aligned}
$$

where $F(x)=K_{2} x_{2}+f\left(x_{1}, x_{2}\right)+T_{\mathrm{d}}+T_{1}+T_{\mathrm{f}}$ is the unknown function which cannot be measured. Thus, we employed the NN to approximate $F(\mathbf{x})$ which can be written as

$$
F(x)=W^{* T} X(\mathbf{x})+\varepsilon
$$

where $\varepsilon$ is the approximation error.

Choose the barrier Lyapunov function as

$$
V_{2}=\frac{J}{2} \log \left(\frac{k_{b 2}^{2}}{k_{b 2}^{2}-z_{2}^{2}}\right)+\frac{1}{2 \Gamma} \tilde{W}^{2}
$$

where $\tilde{W}=W-\widehat{W}$ denotes the estimation error and $\Gamma$ is the design parameter.

Then, differentiating $V_{2}$ yields

$$
\dot{V}_{2}=\frac{z_{2} \dot{z}_{2}}{k_{b 2}^{2}-z_{2}^{2}}+\frac{1}{\Gamma} \tilde{W} \widehat{W}=\frac{z_{2}\left(K_{1} u-F(\mathbf{x})-\dot{\alpha}_{1}\right)}{k_{b 2}^{2}-z_{2}^{2}}+\frac{1}{\Gamma} \tilde{W} \widehat{W}
$$

Using (21), (22) becomes

$$
\begin{aligned}
\dot{V}_{2}= & \frac{1}{k_{b 2}^{2}-z_{2}^{2}} z_{2} K_{1} u-\frac{1}{k_{b 2}^{2}-z_{2}^{2}} z_{2} W^{* T} X(\mathbf{x}) \\
& -\frac{1}{k_{b 2}^{2}-z_{2}^{2}} \varepsilon-\frac{1}{\Gamma} \tilde{W} \dot{\hat{W}} .
\end{aligned}
$$

Using Young's inequality, we obtain

$$
\begin{aligned}
\frac{1}{k_{b 2}^{2}-z_{2}^{2}} z_{2} W^{* T} X(\mathbf{x}) \leq & \frac{1}{2} a^{2}+\frac{1}{2 a^{2}} \frac{1}{\left(k_{b 2}^{2}-z_{2}^{2}\right)^{2}} \\
& \times z_{2}^{2} \bar{W}^{2}\|X(\mathbf{x})\|^{2} \frac{1}{k_{b 2}^{2}-z_{2}^{2}} z_{2} \varepsilon \\
\leq & \frac{1}{2} \frac{1}{\left(k_{b 2}^{2}-z_{2}^{2}\right)^{2}} z_{2}^{2}+\frac{1}{2} \bar{\varepsilon}^{2}
\end{aligned}
$$




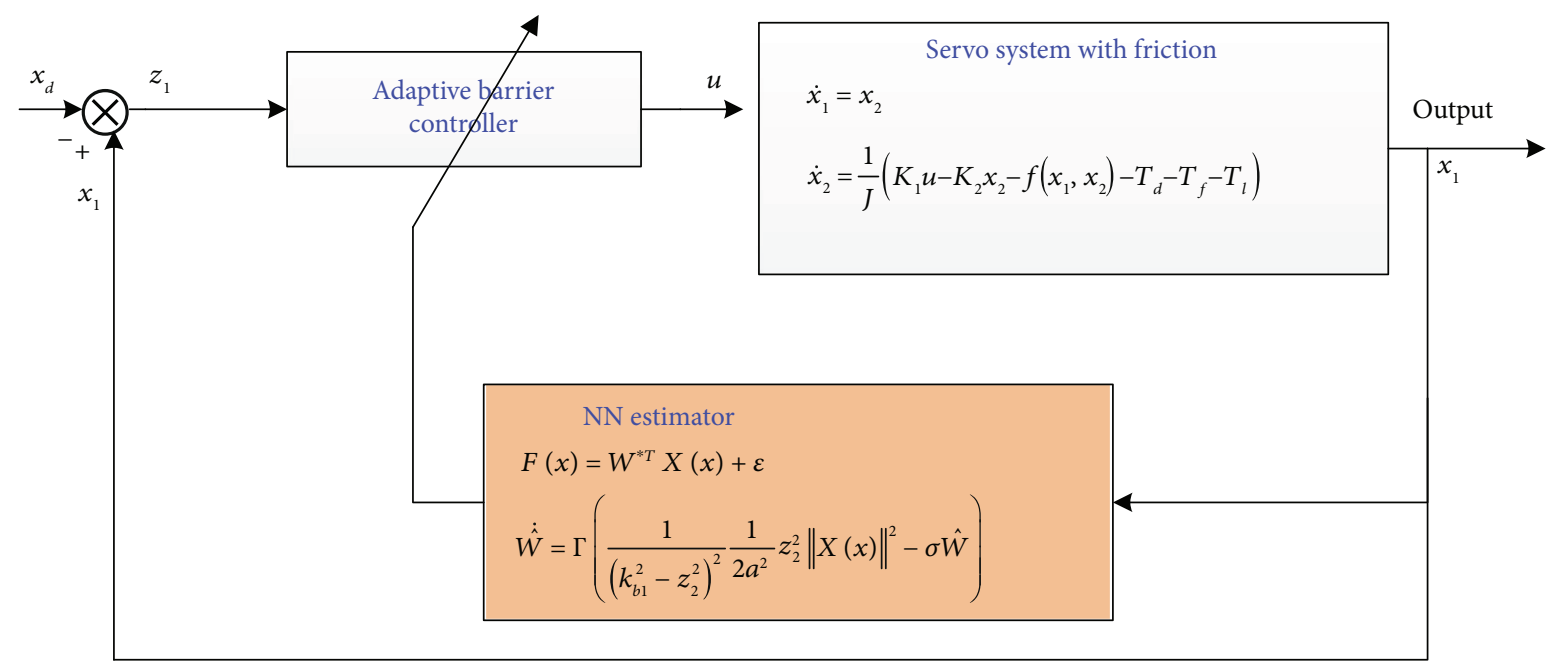

FIgURe 3: Control structure diagram.

where $a$ is a design parameter.

The control signal $u$ can be designed as

$$
u=J\left(-\lambda_{2} z_{2}-\frac{1}{2} \frac{1}{k_{b 2}^{2}-z_{2}^{2}} z_{2}-\frac{1}{2 a^{2}} \frac{1}{k_{b 2}^{2}-z_{2}^{2}} z_{2} \widehat{W}\|X(\mathbf{x})\|^{2}\right),
$$

where $\lambda_{2}$ is a positive constant. The adaptation law $\widehat{W}$ is designed as

$$
\dot{\hat{W}}=\Gamma\left(\frac{1}{\left(k_{b 1}^{2}-z_{2}^{2}\right)^{2}} \frac{1}{2 a^{2}} z_{2}^{2}\|X(\mathbf{x})\|^{2}-\sigma \widehat{W}\right),
$$

where $\Gamma$ and $\sigma$ are the design parameters.

\section{Stability Analysis}

In this section, the stability of the closed-loop system is proven by the Lyapunov stability theory. Based on the design procedure in Section 3, our main result can be summarized in the following theorem.

Theorem 1. Consider servomechanism (1) with Assumption 1. By designing an adaptive controller (26) and virtual controller (17) and choosing the adaptation law (27), it can be guaranteed that all signals of the closed-loop system are semiglobally bounded and the tracking error $z_{1}$ can be kept within a compact set.

Proof 1. Choose a Lyapunov function:

$$
V=V_{1}+V_{2}
$$

Deriving $V$ and substituting (18) and (23) into (28), we have $\dot{V} \leq-\frac{\lambda_{1} z_{1}^{2}}{k_{b 1}^{2}-z_{1}^{2}}-\frac{\lambda_{2} z_{2}^{2}}{k_{b 2}^{2}-z_{2}^{2}}+\frac{z_{1} z_{2}}{k_{b 1}^{2}-z_{1}^{2}}+\frac{1}{2} a^{2}+\frac{1}{2} \bar{\varepsilon}^{2}-\sigma \tilde{W} \widehat{W}$.
Using Young's inequality, one has

$$
-\sigma \tilde{W} \hat{W} \leq-\frac{1}{2} \sigma \tilde{W}^{2}+\frac{1}{2} \sigma W^{2} \frac{z_{1} z_{2}}{k_{b 1}^{2}-z_{1}^{2}} \leq \frac{1}{2} \frac{z_{1}^{2}}{k_{b 1}^{2}-z_{1}^{2}}+\frac{1}{2} z_{2} .
$$

Then, (29) can be written as

$$
\begin{aligned}
\dot{V} \leq & -\frac{\lambda_{1} z_{1}^{2}}{k_{b 1}^{2}-z_{1}^{2}}-\frac{\lambda_{2} z_{2}^{2}}{k_{b 2}^{2}-z_{2}^{2}}+\frac{1}{2} \frac{z_{1}^{2}}{k_{b 1}^{2}-z_{1}^{2}}+\frac{1}{2} z_{2}^{2}+\frac{1}{2} a^{2} \\
& +\frac{1}{2} \bar{\varepsilon}^{2}-\frac{1}{2} \sigma \tilde{W}^{2}+\frac{1}{2} \sigma W^{2} .
\end{aligned}
$$

Let

$$
\begin{aligned}
& \mu=\left\{\frac{\lambda_{1}-1 / 2}{k_{b 1}^{2}-z_{1}^{2}}, \frac{\lambda_{2}}{k_{b 2}^{2}-z_{2}^{2}}-\frac{1}{2}, \frac{1}{2} \sigma \tilde{W}^{2}\right\}, \\
& \varrho=\frac{1}{2} a^{2}+\frac{1}{2} \bar{\varepsilon}^{2}+\frac{1}{2} \sigma W^{2} .
\end{aligned}
$$

From (32), (31) can be represented as

$$
\dot{V}=-\mu V+\varrho .
$$

Multiplying both sides by $e^{\mu t}$, (33) can be written as $d\left(V(t) e^{\mu t}\right) / d t \leq \varrho e^{\mu t}$, and integrating it over $[0, t]$, one has

$$
V(t) \leq\left[V(0)-\frac{\varrho}{\mu}\right] e^{\mu t}+\frac{\varrho}{\mu} \leq V(0)+\frac{\varrho}{\mu} .
$$

From (28) and (34), one can see that $\log k_{b i}^{2} /\left(k_{b i}^{2}-z_{i}^{2}\right)$ and $\tilde{W}, i=1,2$ are bounded. Since $x_{1}=z_{1}+x_{d}$ and $\left|x_{d}\right| \leq$ $A_{0}$, one has $\left|x_{1}\right| \leq\left|z_{1}\right|+\left|x_{d}\right| \leq k_{b 1}+A_{0} \leq k_{c 1}$. Because $\tilde{W}$ and $W$ are bounded, the boundness of $\widehat{W}=\tilde{W}+W$ can be obtained. From $\left|z_{2}\right| \leq k_{b 2}$ and $x_{2}=z_{2}+\alpha_{1}$, one has $\left|x_{2}\right|$ $\leq k_{b 2}+\bar{\alpha}_{1} \leq k_{c 2}$. From (26), one has $\log k_{b i}^{2} /\left(k_{b i}^{2}-z_{i}^{2}\right) \leq[V$ 


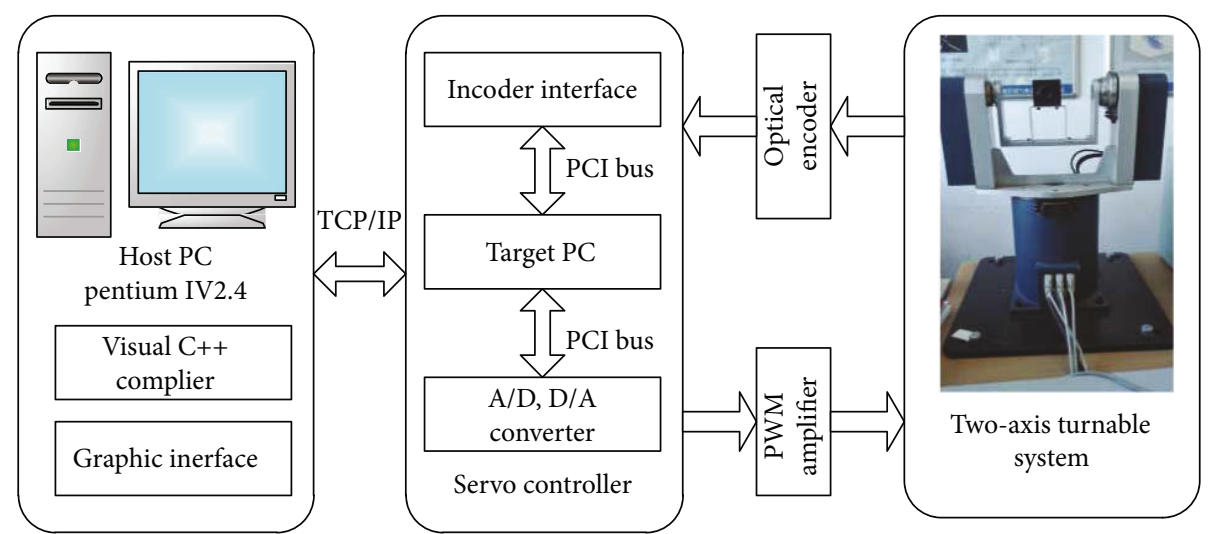

FIgURe 4: Structure of the two-axis servosystem.

$(0)-\mathrm{Q} / \mu] e^{\mu t}+\mathrm{Q} / \mu$. Taking exponentials on both sides of the inequality yields

$$
\frac{k_{b 1}^{2}}{\left(k_{b 1}^{2}-z_{1}^{2}\right)} \leq e^{2[V(0)+\mathrm{\varrho} / \mu] e^{-\mu t}}+2 \frac{\varrho}{\mu} .
$$

From (35), we have $\left|z_{1}\right| \leq k_{b 1}\left(1-e^{-2\left[e^{2[V(0)+\mathrm{e} / \mu] e^{-\mu t}}+2 \mathrm{\varrho} / \mu\right]}\right)^{1 / 2}=$ $\triangle$. If $V(0) \neq \mathrm{Q} / \mu$, it can be concluded that given any $\Delta>k_{b 1}$ $\left(1-e^{-2 \mathrm{\varrho} / \mu}\right)^{1 / 2}$, there exists $T$ such that for any $t>T$, one has $\left|z_{1}\right| \leq \triangle$. As $t \rightarrow \infty,\left|z_{1}\right| \leq k_{b 1}\left(1-e^{-2 \varrho / \mu}\right)^{1 / 2}$. One can see that $z_{1}$ can be made arbitrarily small by choosing the appropriate design parameter.

The proof is completed.

In the following, the tuning guideline for controller parameters is given. The controller parameters include three parts: barrier variables $k_{b 1}$ and $k_{b 2}$ should fulfill the initial conditions; the controller gains $\lambda_{1}$ and $\lambda_{2}$; and the adaptive parameters $\Gamma$, and $\sigma$ should be chosen based on the estimation error. Here, the tuning steps are listed as follows:

(1) Select the adaptive parameters $\Gamma$ and $\sigma$ to make the estimation values to precisely achieve true values.

(2) The barrier parameters $k_{b 1}$ and $k_{b 2}$ can be selected to fulfill with $\left|z_{i}(0)\right|<k_{b i}, i=1,2$.

(3) The feedback term $\lambda_{i} i=1,2$ should be depended on a trial-and-error way to maintain tracking performance and smoothness of the tracking signals. Large gains $\lambda_{i}$ will lead to the vibration.

\section{Experiment Results}

5.1. Experiment Setup. To validate the effectiveness of the developed adaptive barrier control scheme, a turntable servomechanism is used as the experimental platform. The servosystem constituted of servomotor as a controlled plant, a DSP (TMS3202812) connected a personal computer via an A/D converter. The controller is implemented by $\mathrm{C}++$ program on a PC. The sampling time is $0.01 \mathrm{~s}$. In this experiment, the motor position signal is measured by an encoder. The schematic of the servomotion control system is illustrated in Figure 4, and the system parameters are given in Table 1.

5.2. Controller Design. To test the effectiveness of the suggested method. An adaptive neural dynamic surface controller (ANDSC) [62] and PID are employed as a comparison.

(1) Adaptive control (AC): the adaptive control (AC) is developed in this paper. The controller parameters are selected as $\lambda_{1}=8$ and $\lambda_{2}=4$. The barrier function parameters are $k_{b 1}=1.5$ and $k_{b 2}=2$. The NN parameters are chosen as $\Gamma=100$ and $\sigma=0.1$.

(2) ANDSC [62]: the errors are defined as $z_{1}=x_{1}-x_{d}$ and $z_{2}=x_{2}-s_{1}$ with $\mu_{1} \dot{s}_{1}+s_{1}=\alpha_{1}$ and $\alpha_{1}=-k_{1} z_{1}-$ $\widehat{\theta} z_{1} \Phi_{1}^{T} \Phi_{1} / 2-\widehat{\varepsilon}_{1} \tanh \left(z_{1} / \omega_{1}\right)$, and the controller $u$ is $u=-k_{2} z_{2}-\widehat{\theta} z_{2} \Phi_{2}^{T} \Phi_{2} / 2-\widehat{\varepsilon}_{2} \tanh \left(z_{2} / \omega_{2}\right)$ and adaptive laws are $\widehat{\theta}_{i}=\Gamma_{i}\left[z_{i}^{2} \Phi_{i}^{T} \Phi_{i}-\sigma_{i} \widehat{\theta}_{i}\right] / 2$. The controller parameters are $k_{1}=9,, k_{2}=4, \Gamma_{1}=\Gamma_{2}=100, \mu_{1}=$ $0.01, \Gamma_{a 1}=\Gamma_{a 2}=10, \sigma_{1}=\sigma_{a 1}=\sigma_{a 2}=0.01$, and $\omega_{1}=$ $\omega_{2}=1$.

(3) PID: the control law is defined as $u=k_{p}\left(x_{1}-x_{d}\right)+$ $k_{i} \int_{0}^{t}\left(x_{1}-x_{d}\right) d t+k_{d} d\left(x_{1}-x_{d}\right) / d t$, and control gains are chosen as $k_{p}=30, k_{i}=0.05$, and $k_{d}=25$.

\subsection{Experimental Results}

Case 1. To test the effectiveness of the proposed adaptive barrier controller, a sinusoidal signal $x_{d}=0.5 \sin (2 \pi t / 4)$ with amplitude 0.5 and period 4 is adopted in this experiment. The experimental results are shown in Figures 5 and 6. Figure 5 describes the position tracking result for the different controllers (AC, ANDSC, and PID), and Figure 6 gives the tracking error of three controllers. From these figures, we can see that the tracking effectiveness of the proposed adaptive control scheme is better than the other two controllers (e.g., ANDSC and PID). This is mainly because the proposed adaptive controller scheme contains the state constraints and friction compensation. Moreover, ANDSC method produces the smaller tracking error than PID scheme because ANDSC employed NN to compensate for the 
TABLE 1: System parameters.

\begin{tabular}{lc}
\hline Parameters & Quantities \\
\hline$J\left(\mathrm{~kg} \cdot \mathrm{m}^{2}\right)$ & 0.025 \\
$R(\Omega)$ & 10 \\
$L(\mathrm{H})$ & 0.043 \\
$K_{\mathrm{T}}(\mathrm{N} / \mathrm{A})$ & 1.25 \\
$K_{E}(\mathrm{~V} / \mathrm{m} / \mathrm{s})$ & 0.1 \\
\hline
\end{tabular}

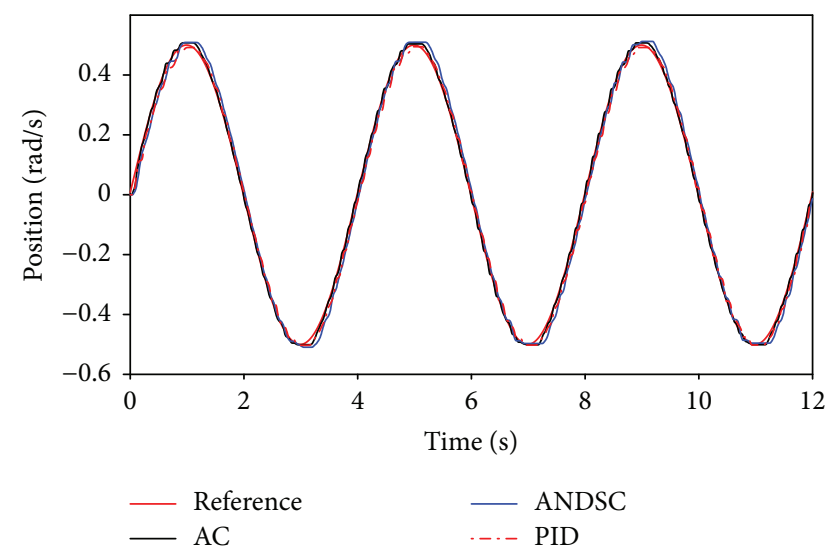

Figure 5: Position tracking for $x_{d}=0.5 \sin (2 \pi t / 4)$.
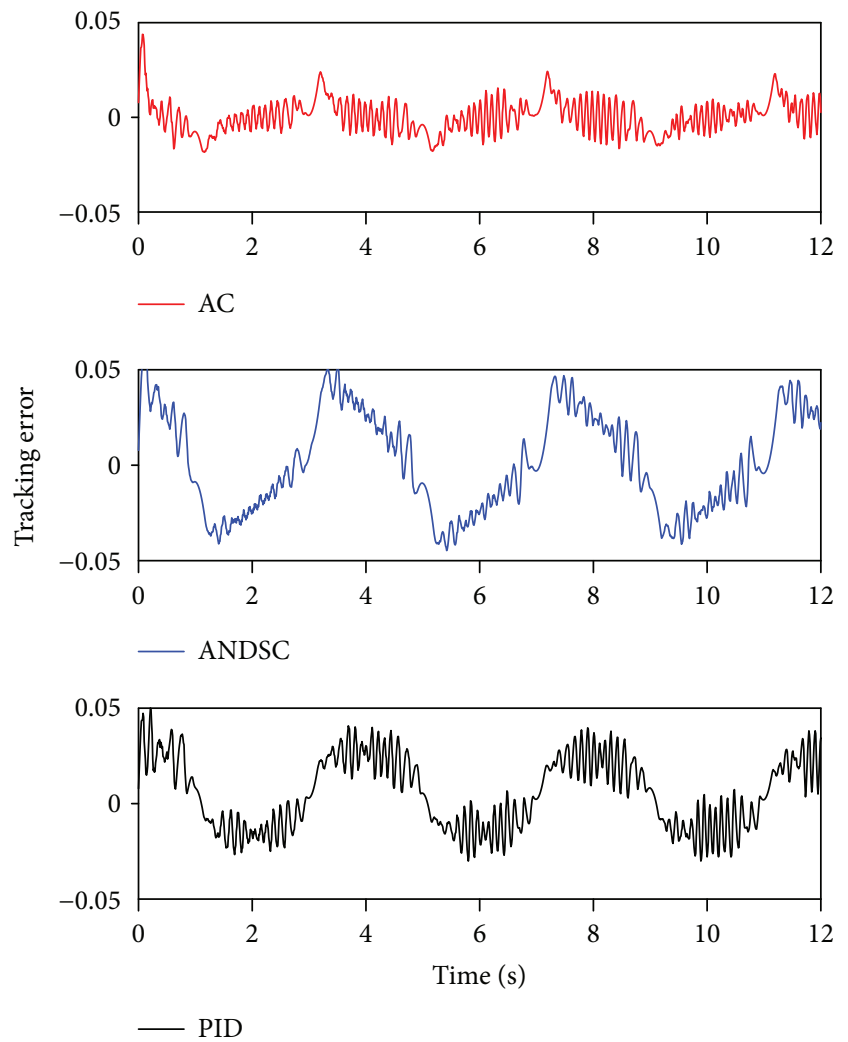

FIgURE 6: Tracking error for $x_{d}=0.5 \sin (2 \pi t / 4)$.

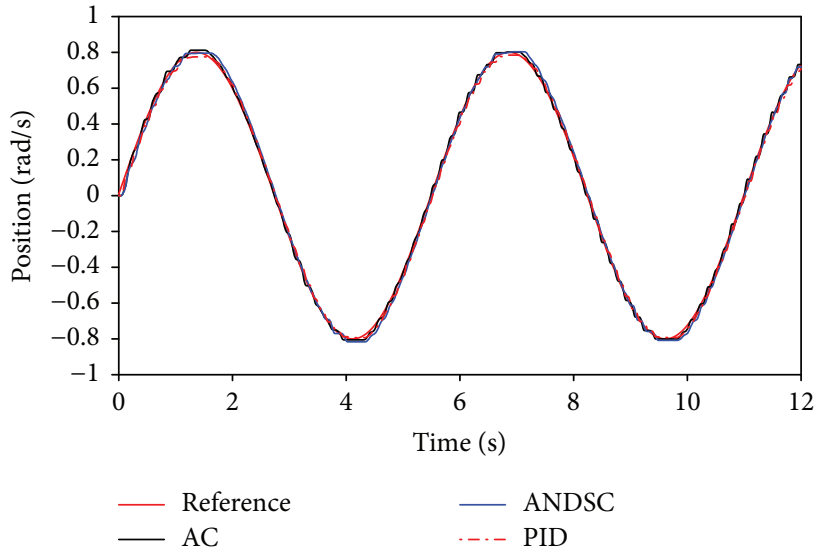

Figure 7: Position tracking for $x_{d}=0.8 \sin (2 \pi t / 5.5)$.

friction dynamics of the servomechanism. Among the three controllers, PID provides the worst tracking performance.

Case 2. To further test the effectiveness of the proposed adaptive controller, another sinusoidal signal $x_{d}=0.8 \sin (2 \pi t / 5.5)$ with large amplitude 0.8 and large period 5.5 is employed in this experiment. The experimental results are depicted in Figures 7 and 8. From these figures, one can find that the proposed adaptive control method provides the best control performance in three controllers. The tracking error is smaller than the ANDSC and PID control schemes. Nevertheless, we may find that the position tracking signal produces fluctuations in comparison to Case 1. This is reasonable because the proposed adaptive controller is able to capture the triggered high-frequency time-varying dynamics and thus to compensate for their effects by calling for corresponding control actions.

\section{Discussion}

All the aforementioned simulation and experiment results show that the control performance of the suggested adaptive control scheme is better than the ANDSC and PID control schemes in terms of the friction dynamics for different trajectories. The reason is that the $\mathrm{NN}$ is employed to estimate the friction dynamics and incorporated into the controller design to compensate for the friction. Thus, the developed adaptive control method is more useful for the position tracking of the servosystem.

\section{Conclusions}

This paper proposed an adaptive barrier controller for servomechanisms with LuGre friction compensation. A modified LuGre friction model is used to capture unknown friction dynamics. Then, the NN is employed to approximate unknown dynamics (i.e., friction, disturbance, and unknown nonlinearities). Moreover, a barrier Lyapunov function (BLF) is introduced to each step in a backstepping design procedure. Then, a novel adaptive control scheme is well suggested to ensure that the full-state constraints are not 

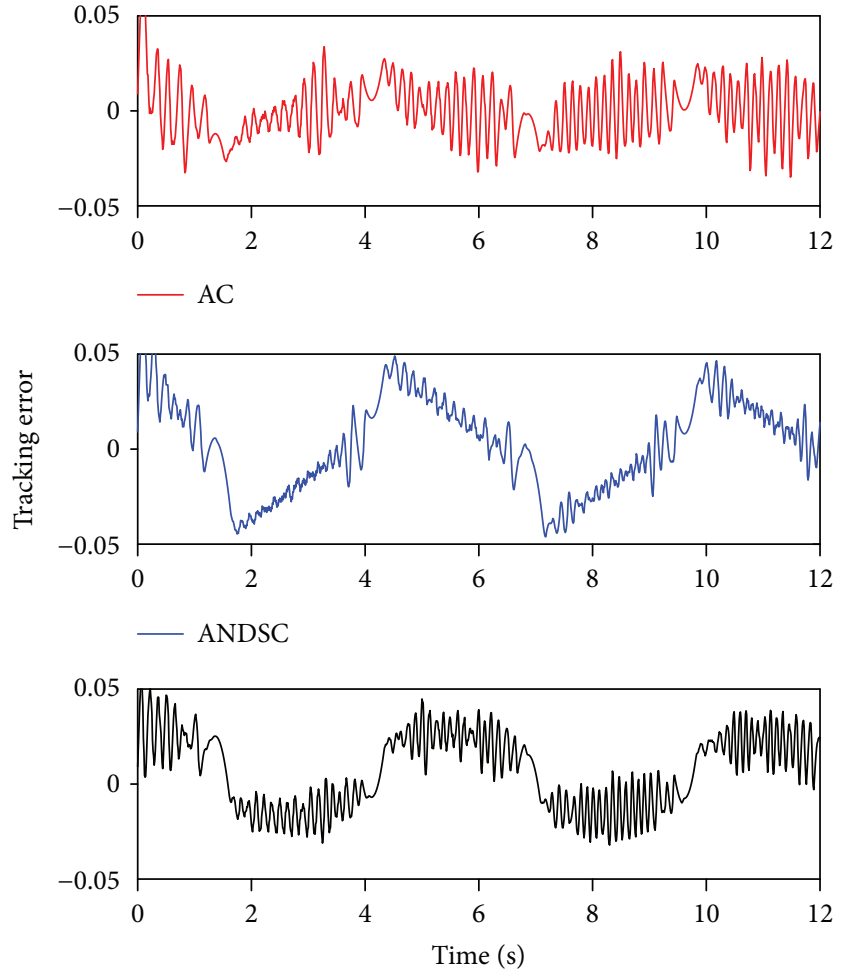

- PID

FIGURE 8: Tracking error for $x_{d}=0.8 \sin (2 \pi t / 5.5)$.

violated. The stability analysis of the design scheme is verified based on the Lyapunov stability theory. Comparative experiments on a turntable servomechanism confirm the effectiveness of the devised control method.

\section{Data Availability}

For data availability, if the researcher needs data of this manuscript, the corresponding author can provide the experiment data.

\section{Conflicts of Interest}

The authors declare that there are no conflicts of interest.

\section{Acknowledgments}

This work is supported by the National Natural Science Foundation of China (61573203), in part by the Natural Science Foundation of Shandong Province under Grant (ZR2018BF022, ZR2016FP10, and ZR2017MF048) and the Scientific Research Foundation of Shandong University of Science and Technology for Recruited Talents (2016RCJJ035).

\section{References}

[1] S. Wang, X. Ren, J. Na, and T. Zeng, "Extended-state-observerbased funnel control for nonlinear servomechanisms with prescribed tracking performance," IEEE Transactions on Automation Science and Engineering, vol. 14, no. 1, pp. 98108, 2017.
[2] J. Na, Q. Chen, X. Ren, and Y. Guo, "Adaptive prescribed performance motion control of servo mechanisms with friction compensation," IEEE Transactions on Industrial Electronics, vol. 61, no. 1, pp. 486-494, 2014.

[3] S. Wang, X. Ren, J. Na, and X. Gao, "Robust tracking and vibration suppression for nonlinear two-inertia system via modified dynamic surface control with error constraint," Neurocomputing, vol. 203, pp. 73-85, 2016.

[4] S. Wang, X. Ren, and J. Na, "Adaptive dynamic surface control based on fuzzy disturbance observer for drive system with elastic coupling," Journal of the Franklin Institute, vol. 353, no. 8, pp. 1899-1919, 2016.

[5] Q. Chen, X. Ren, J. Na, and D. Zheng, "Adaptive robust finitetime neural control of uncertain PMSM servo system with nonlinear dead zone," Neural Computing and Applications, vol. 28, no. 12, pp. 3725-3736, 2017.

[6] J. Wang, F. Wang, Z. Zhang, S. Li, and J. Rodriguez, "Design and implementation of disturbance compensationbased enhanced robust finite control set predictive torque control for induction motor systems," IEEE Transactions on Industrial Informatics, vol. 13, no. 5, pp. 2645-2656, 2017.

[7] H. Yang, J. Sun, Y. Xia, and L. Zhao, "Position control for magnetic rodless cylinders with strong static friction," IEEE Transactions on Industrial Electronics, vol. 65, no. 7, pp. 58065815, 2018.

[8] M. Ruderman and M. Iwasaki, "Sensorless torsion control of elastic-joint robots with hysteresis and friction," IEEE Transactions on Industrial Electronics, vol. 63, no. 3, pp. 18891899, 2016

[9] R. Hao, J. Wang, J. Zhao, and S. Wang, "Observer-based robust control of 6-dof parallel electrical manipulator with fast friction estimation," IEEE Transactions on Automation Science and Engineering, vol. 13, no. 3, pp. 1399-1408, 2016.

[10] J. Yu, P. Shi, W. Dong, and C. Lin, "Adaptive fuzzy control of nonlinear systems with unknown dead zones based on command filtering," IEEE Transactions on Fuzzy Systems, vol. 26, no. 1, pp. 46-55, 2018.

[11] L. Wang, H. Li, Q. Zhou, and R. Lu, “Adaptive fuzzy control for nonstrict feedback systems with unmodeled dynamics and fuzzy dead zone via output feedback," IEEE Transactions on Cybernetics, vol. 47, no. 9, pp. 2400-2412, 2017.

[12] B. Xu, F. Sun, Y. Pan, and B. Chen, "Disturbance observer based composite learning fuzzy control of nonlinear systems with unknown dead zone," IEEE Transactions on Systems, Man, and Cybernetics: Systems, vol. 47, no. 8, pp. 1854-1862, 2017.

[13] H. Du, X. Chen, G. Wen, X. Yu, and J. Lu, "Discrete-time fast terminal sliding mode control for permanent magnet linear motor," IEEE Transactions on Industrial Electronics, no. 99, p. $1,2018$.

[14] D. Won, W. Kim, and M. Tomizuka, "High-gain-observerbased integral sliding mode control for position tracking of electrohydraulic servo systems," IEEE/ASME Transactions on Mechatronics, vol. 22, no. 6, pp. 2695-2704, 2017.

[15] W. Zhao, X. Ren, and S. Wang, "Parameter estimation-based timevarying sliding mode control for multimotor driving servo systems," IEEE/ASME Transactions on Mechatronics, vol. 22, no. 5, pp. 2330-2341, 2017.

[16] L. Zhao, J. Yu, C. Lin, and H. Yu, "Distributed adaptive fixedtime consensus tracking for second-order multi-agent systems 
using modified terminal sliding mode," Applied Mathematics and Computation, vol. 312, pp. 23-35, 2017.

[17] S. Ding, L. Liu, and W. X. Zheng, "Sliding mode direct yawmoment control design for in-wheel electric vehicles," IEEE Transactions on Industrial Electronics, vol. 64, no. 8, pp. 6752-6762, 2017.

[18] S. Ding, W. X. Zheng, J. Sun, and J. Wang, "Second-order sliding mode controller design and its implementation for buck converters," IEEE Transactions on Industrial Informatics, vol. 14, no. 5, pp. 1990-2000, 2018.

[19] J. Yu, P. Shi, W. Dong, B. Chen, and C. Lin, "Neural networkbased adaptive dynamic surface control for permanent magnet synchronous motors," IEEE Transactions on Neural Networks and Learning Systems, vol. 26, no. 3, pp. 640-645, 2015.

[20] J. Yao, W. Deng, and W. Sun, "Precision motion control for electro-hydraulic servo systems with noise alleviation: a desired compensation adaptive approach," IEEE/ASME Transactions on Mechatronics, vol. 22, no. 4, pp. 1859$1868,2017$.

[21] R. E. Precup, R. C. David, E. M. Petriu, M. B. Radac, and S. Preitl, "Adaptive gsa-based optimal tuning of pi controlled servo systems with reduced process parametric sensitivity, robust stability and controller robustness," IEEE Transactions on Cybernetics, vol. 44, no. 11, pp. 1997-2009, 2014.

[22] Y. Zhou, Q. Zhang, H. Wang, P. Zhou, and T. Chai, "Ekf-based enhanced performance controller design for nonlinear stochastic systems," IEEE Transactions on Automatic Control, vol. 63, no. 4, pp. 1155-1162, 2018.

[23] Q. Zhang, J. Zhou, H. Wang, and T. Chai, “Output feedback stabilization for a class of multi-variable bilinear stochastic systems with stochastic coupling attenuation," IEEE Transactions on Automatic Control, vol. 62, no. 6, pp. 2936-2942, 2017.

[24] J. Yu, P. Shi, W. Dong, and C. Lin, "Command filtering-based fuzzy control for nonlinear systems with saturation input," IEEE Transactions on Cybernetics, vol. 47, no. 9, pp. 24722479, 2017.

[25] X. Xie, D. Yue, J. H. Park, and H. Li, "Relaxed fuzzy observer design of discrete-time nonlinear systems via two effective technical measures," IEEE Transactions on Fuzzy Systems, p. 1, 2018.

[26] J. Yu, P. Shi, W. Dong, and H. Yu, "Observer and commandfilter-based adaptive fuzzy output feedback control of uncertain nonlinear systems," IEEE Transactions on Industrial Electronics, vol. 62, no. 9, pp. 5962-5970, 2015.

[27] L. Zhao, J. Yu, C. Lin, and Y. Ma, "Adaptive neural consensus tracking for nonlinear multiagent systems using finite-time command filtered backstepping," IEEE Transactions on Systems, Man, and Cybernetics: Systems, pp. 1-10, 2017.

[28] S. Wang, J. Na, and X. Ren, "Rise-based asymptotic prescribed performance tracking control of nonlinear servo mechanisms," IEEE Transactions on Systems, Man, and Cybernetics: Systems, pp. 1-12, 2017.

[29] Q. Chen, L. Shi, J. Na, X. Ren, and Y. Nan, “Adaptive echo state network control for a class of pure-feedback systems with input and output constraints," Neurocomputing, vol. 275, pp. 1370-1382, 2018.

[30] J. Na, A. S. Chen, G. Herrmann, R. Burke, and C. Brace, "Vehicle engine torque estimation via unknown input observer and adaptive parameter estimation," IEEE Transactions on Vehicular Technology, vol. 67, no. 1, pp. 409-422, 2018.
[31] B. K. Ohnishi, "A new servo method in mechatronics trans," Japanese Society of Electrical Engineering, vol. 177, pp. 83-86, 1987.

[32] K.-H. Kim and M.-J. Youn, "A nonlinear speed control for a pm synchronous motor using a simple disturbance estimation technique," IEEE Transactions on Industrial Electronics, vol. 49, no. 3, pp. 524-535, 2002.

[33] W.-H. Chen, D. J. Ballance, P. J. Gawthrop, and J. O'Reilly, “A nonlinear disturbance observer for robotic manipulators," IEEE Transactions on Industrial Electronics, vol. 47, no. 4, pp. 932-938, 2000.

[34] J. Han, "From PID to active disturbance rejection control," IEEE Transactions on Industrial Electronics, vol. 56, no. 3, pp. 900-906, 2009.

[35] R. Cui, L. Chen, C. Yang, and M. Chen, "Extended state observer-based integral sliding mode control for an underwater robot with unknown disturbances and uncertain nonlinearities," IEEE Transactions on Industrial Electronics, vol. 64, no. 8, pp. 6785-6795, 2017.

[36] W. Xue, W. Bai, S. Yang, K. Song, Y. Huang, and H. Xie, “Adrc with adaptive extended state observer and its application to air fuel ratio control in gasoline engines," IEEE Transactions on Industrial Electronics, vol. 62, no. 9, pp. 5847-5857, 2015.

[37] Z. Zhu, D. Xu, J. Liu, and Y. Xia, "Missile guidance law based on extended state observer," IEEE Transactions on Industrial Electronics, vol. 60, no. 12, pp. 5882-5891, 2013.

[38] H. Liu and S. Li, "Speed control for PMSM servo system using predictive functional control and extended state observer," IEEE Transactions on Industrial Electronics, vol. 59, no. 2, pp. 1171-1183, 2012.

[39] S. Li and Z. Liu, "Adaptive speed control for permanentmagnet synchronous motor system with variations of load inertia," IEEE Transactions on Industrial Electronics, vol. 56, no. 8, pp. 3050-3059, 2009.

[40] J. Yang, J. Wang, B. Wu, S. Li, and Q. Li, "Extended state observer-based sliding mode control for PWM-based DCDC buck power converter systems with mismatched disturbances," IET Control Theory \& Applications, vol. 9, no. 4, pp. 579-586, 2015.

[41] S. E. Talole, J. P. Kolhe, and S. B. Phadke, "Extended-stateobserver-based control of flexible-joint system with experimental validation," IEEE Transactions on Industrial Electronics, vol. 57, no. 4, pp. 1411-1419, 2010.

[42] D. Shi, J. Xue, L. Zhao, J. Wang, and Y. Huang, "Event-triggered active disturbance rejection control of DC torque motors," IEEE/ASME Transactions on Mechatronics, vol. 22, no. 5, pp. 2277-2287, 2017.

[43] C. P. Bechlioulis and G. A. Rovithakis, "Robust adaptive control of feedback linearizable MIMO nonlinear systems with prescribed performance," IEEE Transactions on Automatic Control, vol. 53, no. 9, pp. 2090-2099, 2008.

[44] C. P. Bechlioulis and G. A. Rovithakis, "Adaptive control with guaranteed transient and steady state tracking error bounds for strict feedback systems," Automatica, vol. 45, no. 2, pp. 532-538, 2009.

[45] J. Na, Y. Huang, X. Wu, G. Gao, G. Herrmann, and J. Z. Jiang, "Active adaptive estimation and control for vehicle suspensions with prescribed performance," IEEE Transactions on Control Systems Technology, pp. 1-15, 2017.

[46] J. Na, G. Herrmann, and K. Zhang, "Improving transient performance of adaptive control via a modified reference model 
and novel adaptation," International Journal of Robust and Nonlinear Control, vol. 27, no. 8, pp. 1351-1372, 2017.

[47] S. L. Dai, S. He, H. Lin, and C. Wang, "Platoon formation control with prescribed performance guarantees for USVS," IEEE Transactions on Industrial Electronics, vol. 65, no. 5, pp. 42374246, 2018.

[48] C. P. Bechlioulis and G. A. Rovithakis, "Robust partial-state feedback prescribed performance control of cascade systems with unknown nonlinearities," IEEE Transactions on Automatic Control, vol. 56, no. 9, pp. 2224-2230, 2011.

[49] J. Na, M. N. Mahyuddin, G. Herrmann, X. Ren, and P. Barber, "Robust adaptive finite time parameter estimation and control for robotic systems," International Journal of Robust and Nonlinear Control, vol. 25, no. 16, pp. 3045-3071, 2015.

[50] J. X. Zhang and G. H. Yang, "Prescribed performance faulttolerant control of uncertain nonlinear systems with unknown control directions," IEEE Transactions on Automatic Control, vol. 62, no. 12, pp. 6529-6535, 2017.

[51] M. Wang and A. Yang, "Dynamic learning from adaptive neural control of robot manipulators with prescribed performance," IEEE Transactions on Systems, Man, and Cybernetics: Systems, vol. 47, no. 8, pp. 2244-2255, 2017.

[52] B. Ren, S. S. Ge, K. P. Tee, and T. H. Lee, "Adaptive neural control for output feedback nonlinear systems using a barrier Lyapunov function," IEEE Transactions on Neural Networks, vol. 21, no. 8, pp. 1339-1345, 2010.

[53] W. He, C. Sun, and S. S. Ge, "Top tension control of a flexible marine riser by using integral-barrier Lyapunov function," IEEE/ASME Transactions on Mechatronics, vol. 20, no. 2, pp. 497-505, 2015.

[54] Y. J. Liu, J. Li, S. Tong, and C. L. P. Chen, "Neural network control-based adaptive learning design for nonlinear systems with full-state constraints," IEEE Transactions on Neural Networks and Learning Systems, vol. 27, no. 7, pp. 1562-1571, 2016.

[55] B. Xu, Z. Shi, F. Sun, and W. He, "Barrier Lyapunov function based learning control of hypersonic flight vehicle with AOA constraint and actuator faults," IEEE Transactions on Cybernetics, pp. 1-11, 2018.

[56] Y. Liu, J. Yu, H. Yu, C. Lin, and L. Zhao, "Barrier Lyapunov functions-based adaptive neural control for permanent magnet synchronous motors with full-state constraints," IEEE Access, vol. 5, pp. 10382-10389, 2017.

[57] Z. Chen, Z. Li, and C. L. P. Chen, "Adaptive neural control of uncertain MIMO nonlinear systems with state and input constraints," IEEE Transactions on Neural Networks and Learning Systems, vol. 28, pp. 1318-1330, 2017.

[58] J. Yao, W. Deng, and Z. Jiao, "Adaptive control of hydraulic actuators with LuGre model-based friction compensation," IEEE Transactions on Industrial Electronics, vol. 62, no. 10, pp. 6469-6477, 2015.

[59] D. Zheng, W. F. Xie, X. Ren, and J. Na, "Identification and control for singularly perturbed systems using multitimescale neural networks," IEEE Transactions on Neural Networks and Learning Systems, vol. 28, no. 2, pp. 321-333, 2017.

[60] J. Na, X. Ren, and D. Zheng, "Adaptive control for nonlinear pure-feedback systems with high-order sliding mode observer," IEEE Transactions on Neural Networks and Learning Systems, vol. 24, no. 3, pp. 370-382, 2013.
[61] G. Sun, D. Li, and X. Ren, "Modified neural dynamic surface approach to output feedback of MIMO nonlinear systems," IEEE Transactions on Neural Networks and Learning Systems, vol. 26, no. 2, pp. 224-236, 2015.

[62] J. Na, X. Ren, G. Herrmann, and Z. Qiao, "Adaptive neural dynamic surface control for servo systems with unknown dead-zone," Control Engineering Practice, vol. 19, no. 11, pp. 1328-1343, 2011. 


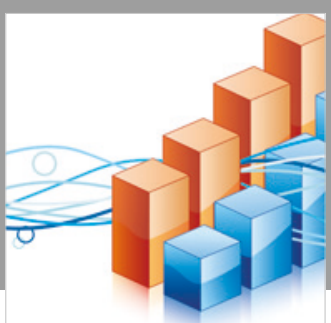

Advances in

Operations Research

\section{-n-m}
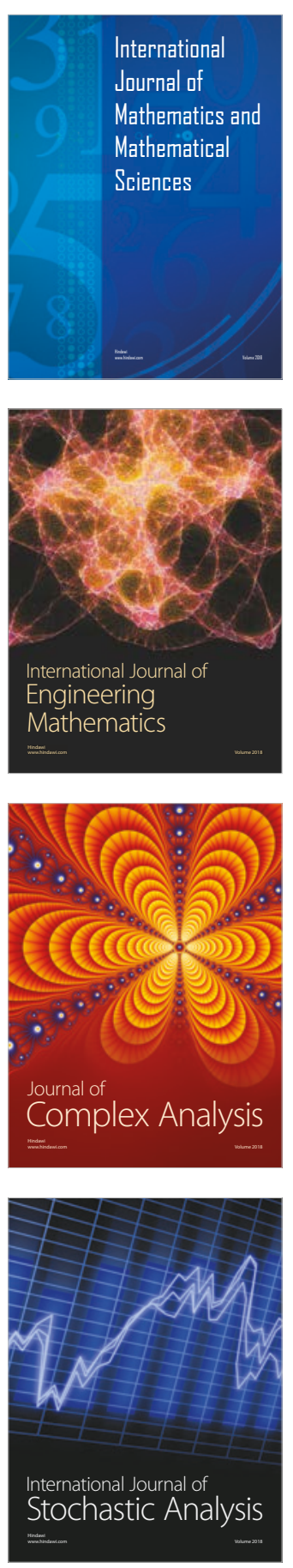
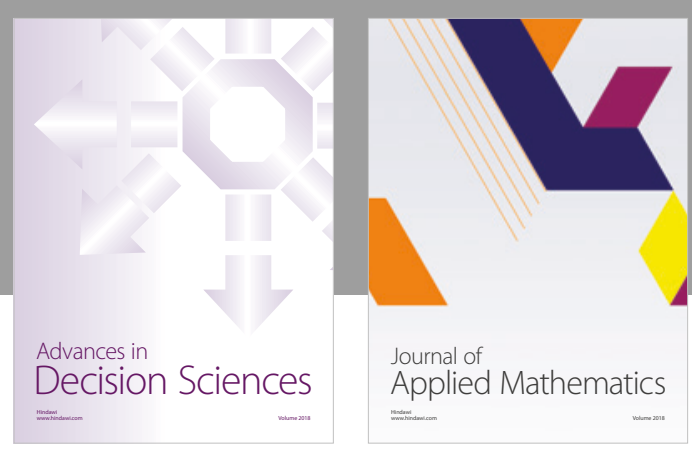

Journal of

Applied Mathematics
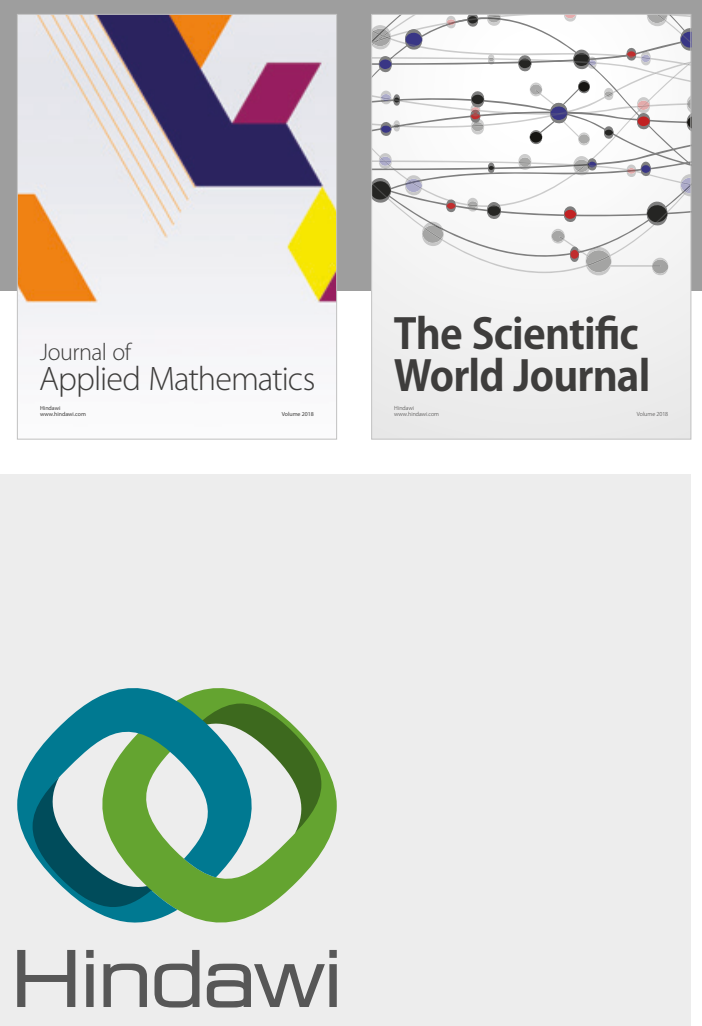

Submit your manuscripts at

www.hindawi.com

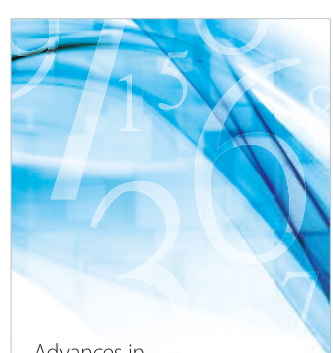

Advances in
Numerical Analysis
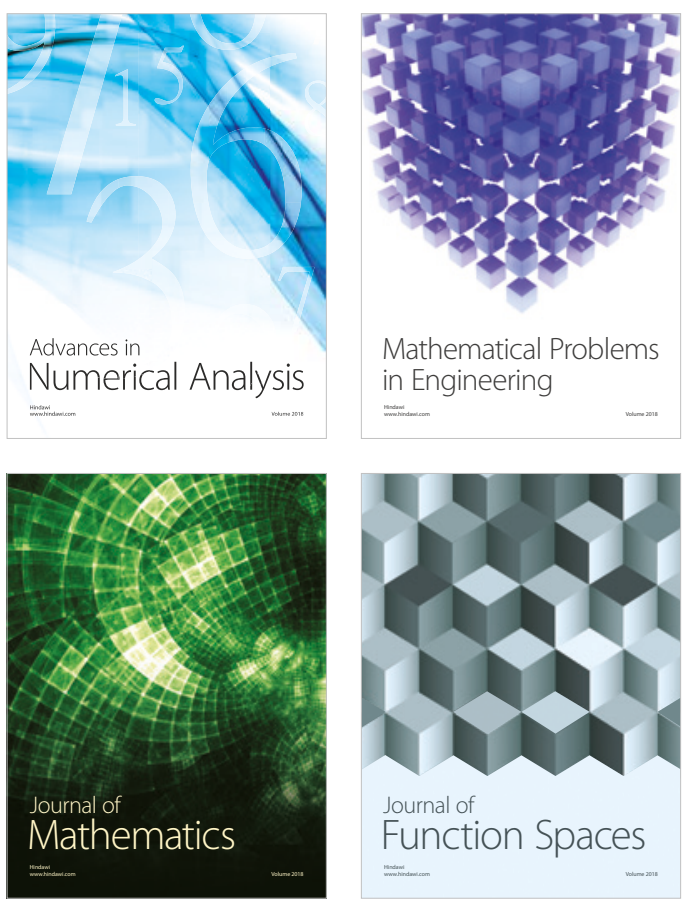

Mathematical Problems in Engineering

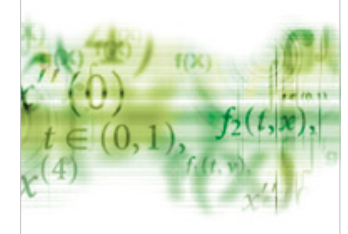

International Journal of

Differential Equations

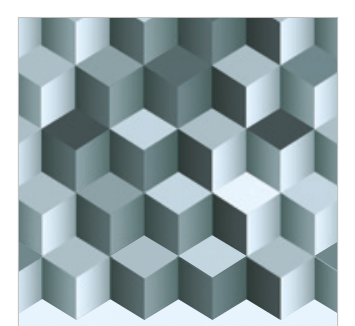

Journal of

Function Spaces
The Scientific

World Journal

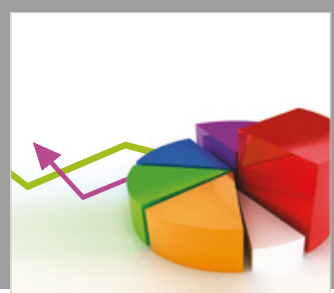

Journal of

Probability and Statistics
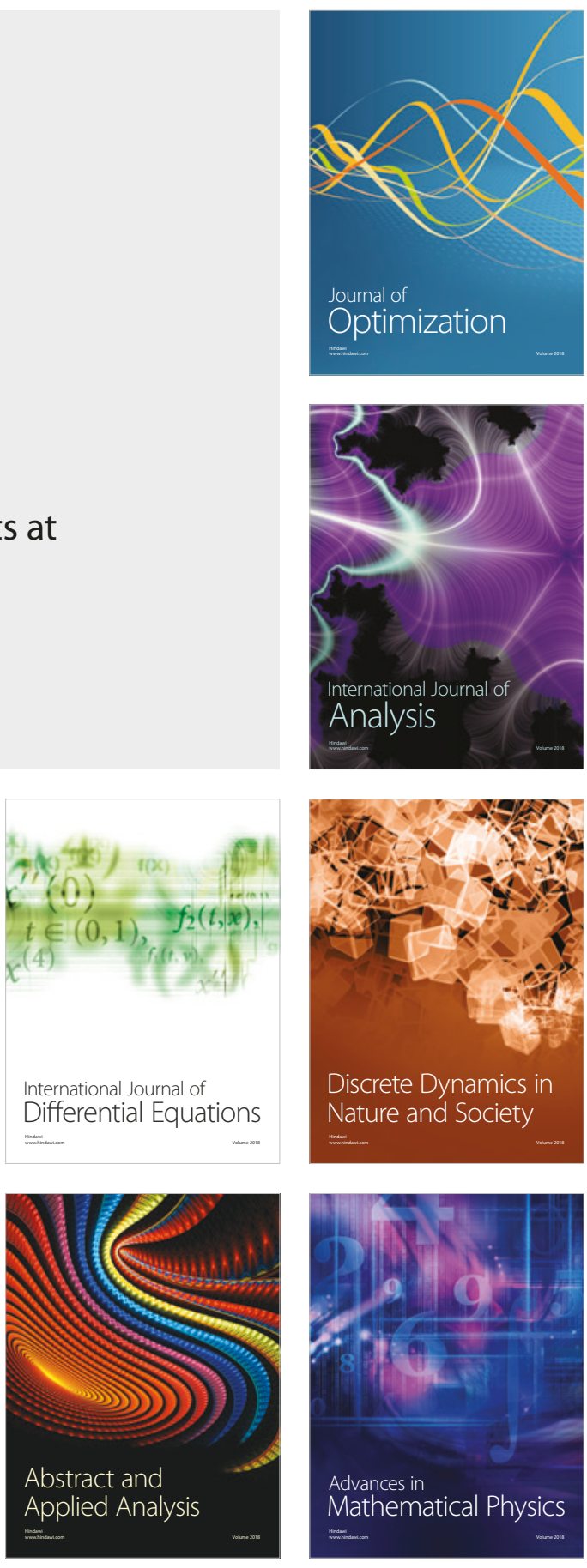